\title{
NOUVELle
}

\section{Des neuropeptides impliqués dans la morphogenèse de l'axe vertébral}

\section{Les leçons du poisson et du crapaud}

Hervé Tostivint ${ }^{1}$, Faredin Alejevski ${ }^{1}$, Michelle Leemans ${ }^{1}$, Anne-Laure Gaillard ${ }^{1}$, Sébastien Le Mével ${ }^{1}$, Anthony Herrel ${ }^{2}$, Jean-Baptiste Fini ${ }^{1}$, Guillaume Pézeron ${ }^{1}$

> Urpl et Urp2, deux neuropeptides de la famille de l'urotensine 2 (Urp pour urotensin-related peptides), une hormone apparentée à la somatostatine, ont été découverts chez l'anguille et le poisson-zèbre, respectivement [1]. Chez le poisson-zèbre, ils sont produits principalement par des neurones particuliers situés le long du canal central de la moelle épinière, qui sont appelés, pour cette raison, cerebrospinal fluidcontacting neurons (CSF-cN) $[2,3]$. Le rôle de ces deux peptides n'a été révélé qu'en 2018, par une équipe sino-singapourienne qui a rapporté que l'utilisation, chez le poisson-zèbre, de morpholinos destinés à bloquer leur synthèse causait une courbure anormale de l'axe des embryons [4]. Nous avons, quant à nous, observé que la double invalidation des gènes codant Urpl et Urp2 provoquait des déformations marquées de la colonne vertébrale. Ces déformations apparaissent toutefois un peu plus tard qu'avec les morpholinos, durant la phase larvaire, et s'aggravent chez le poisson adulte. L'invalidation du gène codant le récepteur Utr4, I'un des récepteurs des peptides de la famille de l'urotensine 2, spécifiquement exprimé dans les myotomes dorsaux, provoque les mêmes défauts [4] (Figure la), ce qui suggère que les deux peptides exercent leurs effets par l'intermédiaire de ce récepteur. Ces travaux ont donc permis de mettre en évidence le rôle d'une nouvelle voie de signalisation dans la formation de l'axe vertébral des poissons. On peut penser que les peptides Urpl et Urp2 participent, par cette voie, au contrôle de la contraction de la musculature du tronc, et garantissent ainsi la rectitude de l'axe vertébral au cours du développement. Cette découverte a suscité un grand intérêt dans la communauté scientifique, car les déformations de l'axe vertébral observées chez les poissons mutants évoquent certaines des manifestations de la scoliose dans l'espèce humaine. Or le poisson-zèbre
'Physiologie moléculaire et adaptation, CNRS UMR 7221, Muséum national d'histoire naturelle, Paris, France.

${ }^{2}$ Mécanismes adaptatifs et évolution, CNRS UMR 7179, Muséum national d'histoire naturelle, Paris, France.

herve.tostivint@mnhn.fr

guillaume.pezeron@mnhn.fr

s'est révélé un modèle de choix pour la recherche des causes de la scoliose [5]. Sans surprise, l'étude de Zhang et al. [4] a donc été suivie de plusieurs autres, précisant notamment les conditions de sécrétion de Urpl et de Urp2 par les neurones qui les synthétisent. Ainsi, il a été montré que cette sécrétion dépendait de l'intégrité de la fibre de Reissner, une structure fibreuse présente dans la lumière de la moelle épinière et en contact étroit avec ces neurones, et qu'elle pouvait être activée par la noradrénaline $[4,6,7]$.

Malgré l'intérêt des résultats obtenus chez le poisson, leur extrapolation à notre espèce est difficile, car ni les peptides Urpl et Urp2, ni leur récepteur, Utr4, n'existent chez les mammifères. Cependant, l'étude que nous venons de réaliser chez le xénope, un crapaud d'origine africaine, permet de mieux évaluer leur portée [8]. Nous avons identifié les gènes codant le récepteur Utr4 et son ligand Urp2 dans cette 


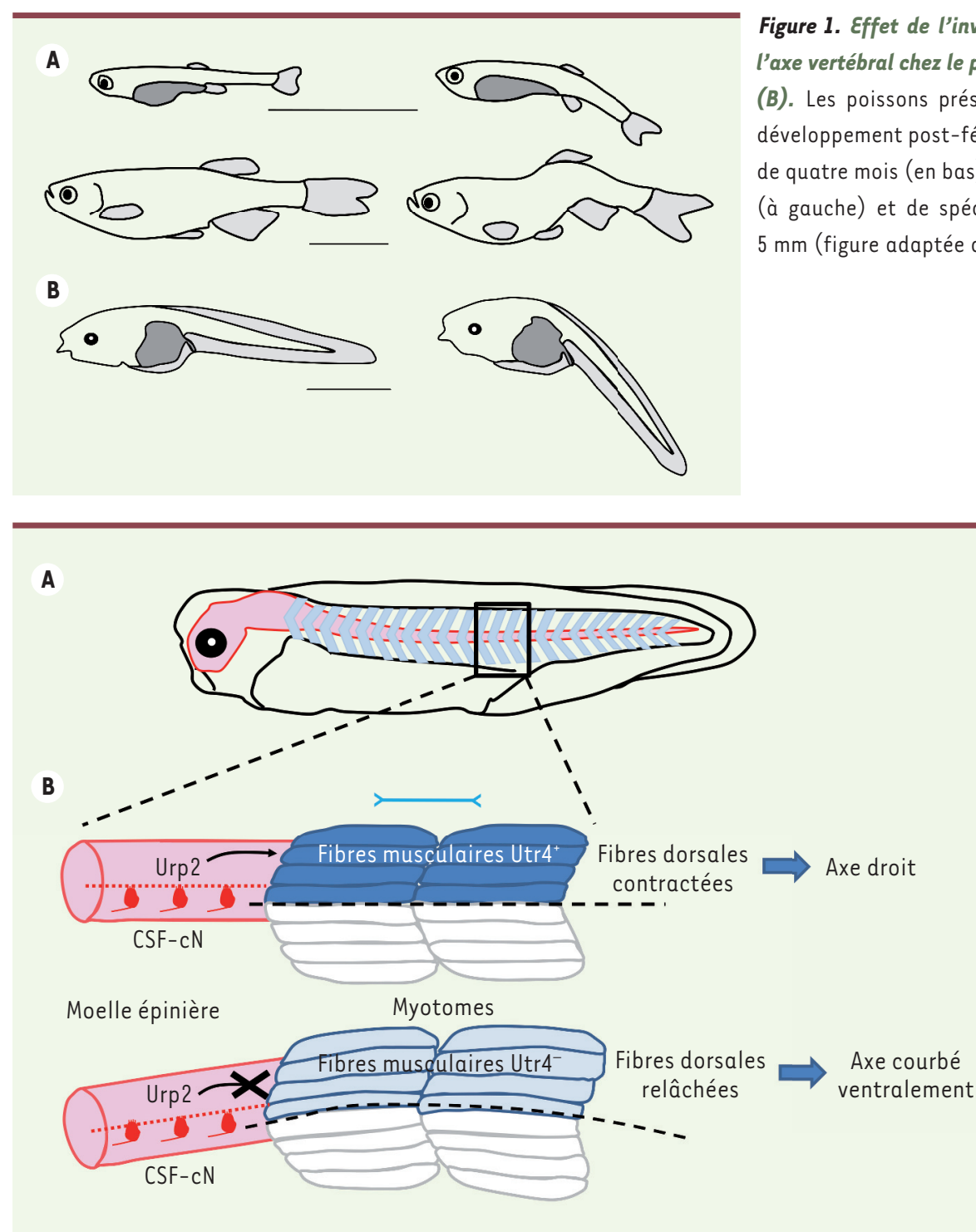

Figure 2. Modèle d'action de la voie Urp4 sur la formation de l'axe vertébral. A. Têtard de xénope à un stade où commencent à s'exprimer le récepteur Utr4 et son ligand Urp2 (stade un peu plus précoce que celui du spécimen présenté dans la figure 1). Le système nerveux est figuré en rose, et les myotomes en bleu. $B$. Détail de la région du tronc encadrée en $\boldsymbol{A}$. Chez les têtards témoins (en haut), la contraction des fibres musculaires $\left(U \operatorname{tr} 4^{+}\right)$de la partie dorsale des myotomes activée par la voie Utr4 assure un tonus qui permet à l'axe vertébral de se former normalement. Chez les têtards dont le gène utr4 a été invalidé (en bas), ces fibres musculaires (Utr4-) ne permettent plus de maintenir ce tonus. Le déséquilibre des forces musculaires exercées sur l'axe vertébral conduit à sa courbure ventrale. La double flèche illustre l'orientation des fibres musculaires au sein des myotomes, et les effets de leur raccourcissement. CSF-cN : cerebro-spinal fluid-contacting neurons; $c c$ : canal central de la moelle épinière.

espèce, et montré qu'ils sont exprimés dans les mêmes types cellulaires que chez le poisson-zèbre, i.e. la musculature dorsale du tronc pour le premier, et les neurones CSF-cN spinaux pour le second. En revanche, nous n'avons pas trouvé trace du gène codant le peptide Urpl, qui a donc vraisemblablement été perdu au cours de l'évolution des amphibiens (auquel le xénope est rattaché). Afin de tester la fonctionnalité de la voie Utr4 chez le xénope, nous avons muté le gène codant ce récepteur, par la technique CRISPR/Cas9, dans des œufs qui venaient d'être fécondés. Nous avons alors observé que les têtards qui en étaient issus développaient rapidement une queue anormalement courbée (Figure Ib). Comme chez le poissonzèbre, la courbure anormale de l'axe observée chez les mutants peut être interprétée comme le résultat d'un relâchement des fibres des myotomes dorsaux (Figure 2).

Nos travaux montrent donc que la voie de signalisation impliquant le récepteur Utr4, que l'on pensait jusqu'à présent restreinte aux seuls poissons, existe aussi chez des tétrapodes et y joue un rôle apparemment semblable. Compte tenu des relations phylogénétiques entre les poissons et les amphibiens, ces résultats suggèrent que la voie impliquant Utr4 constitue un mécanisme conservé de contrôle de la morphogenèse de l'axe des vertébrés, vraisemblablement apparu, au plus tard, au moment de l'émergence des premiers vertébrés à squelette osseux, il y a plus de 400 millions d'années. Puisque les composants majeurs de la voie Utr4 sont absents chez les mammifères, on suppose qu'ils 
ont été perdus au cours de l'évolution. La question est donc de savoir si une voie équivalente à celle des poissons et des amphibiens, mais reposant sur d'autres acteurs moléculaires, existe encore chez les mammifères. Les travaux d'une équipe chinoise semblent accréditer cette hypothèse, puisqu'ils ont révélé l'existence de mutations dans le gène codant Utrl, un récepteur apparenté à Utr4, chez des individus atteints de scoliose [9]. Dans la mesure où aucun défaut de l'axe vertébral n'a pour l'instant été rapporté chez les souris invalidées pour le gène codant Utrl [10], des recherches complémentaires sont cependant nécessaires pour répondre à la question.

En conclusion, ces travaux montrent l'intérêt du modèle poisson-zèbre pour la recherche des causes de la scoliose humaine, mais aussi ses limites, inci- tant à recourir à une plus grande diversité d'espèces modèles afin de tirer le meilleur parti de l'approche évolutionniste. $\diamond$

Neuropeptides involved in proper spine morphogenesis: Lessons from fish and toad

\section{LIENS D'INTÉRÊT}

Les auteurs déclarent n'avoir aucun lien d'intérêt concernant les données publiées dans cet article.

\section{RÉFÉRENCES}

1. Tostivint $H, O$ campo Daza D, Bergqvist CA, et al. Molecular evolution of GPCRs: Somatostatin/ urotensin II receptors. J Mol Endocrinol 2014 ; 52 : T61-86.

2. Parmentier C, Hameury $\varepsilon$, Dubessy $C$, et al. Occurrence of two distinct urotensin II-related peptides in zebrafish provides new insight into the evolutionary history of the urotensin II gene family. Endocrinology $2011 ; 152: 2330-41$.

3. Quan FB, Dubessy C, Galant S, et al. Comparative distribution and in vitro activities of the urotensin II-related peptides URPI and URP2 in zebrafish: evidence for their colocalization in spinal cerebrospinal fluid-contacting neurons. PloS One $2015 ; 10$ : e0119290.

4. Zhang X, Jia S, Chen Z, et al. Cilia-driven cerebrospinal fluid flow directs expression of urotensin neuropeptides to straighten the vertebrate body axis. Nat Genet 2018 ; 50 : 1666-73.

5. Boswell CW, Ciruna B. Understanding idiopathic scoliosis: A new zebrafish school of thought. Trends Genet 2017 ; 33 : 183-96.

6. Lu H, Shagirova A, Goggi JL, et al. Reissner fibreinduced urotensin signalling from cerebrospinal fluid-contacting neurons prevents scoliosis of the vertebrate spine. Biol Open 2020 ; 9 : bio052027.

7. Cantaut-Belarif $y$, Orts Del'Immagine A, Penru M, et al. Adrenergic activation modulates the signal from the Reissner fiber to cerebrospinal fluid-contacting neurons during development. elife 2020 ; 9 : e59469.

8. Alejevski F, Leemans M, Gaillard AL, et al. Conserved role of the urotensin II receptor 4 signalling pathway to control body straightness in a tetrapod. Open Biol $2021 ; 11: 210065$.

9. Dai Z, Wang Y, Wu Z, et al. Novel mutations in UTS2R are associated with adolescent idiopathic scoliosis in the Chinese population. Spine 2021 ; 46 : ع288-93.

10. Behm DJ, Harrison SM, Ao Z, et al. Deletion of the UT receptor gene results in the selective loss of urotensin-II contractile activity in aortae isolated from UT receptor knockout mice. Br J Pharmacol $2003 ; 139: 464-72$ 